\title{
Prime Decomposition Encoding: An Analytical Tool by the Use of Arithmetic Mapping of Drum-Set Timelines
}

\author{
${ }^{*}$ Carlos Mathitas \\ Fluminense Federal University \\ prof carlosmathias@gmail.com \\ Orcid: 0000-0001-7949-2324 \\ Carlos Almada \\ Federal University of Rio de Janeiro \\ carlosalmada@musica.ufrj.br \\ Orcid: 0000-0001-5888-2868 \\ DOI: $10.46926 /$ musmat. 2021v5n2.41-64
}

\begin{abstract}
This paper introduces an original proposal intended to encode timelines as univocal integers, by the use of arithmetic mapping, exploring inherent properties of prime numbers. A group of algorithms were developed in order to encode drum-set timelines (as well as to retrieve the original rhythms from the codes), either individually or taken together, forming grooves. Additional parameters (dynamic levels and timbre) are also included in the encoding process. Geometrical representation of the grooves, adopting terminology and methodology proposed by Gottfried Toussaint[19], is also provided. Some practical application, addressing analysis and composition, are suggested at the last section of the study.
\end{abstract}

Keywords: Fundamental Theorem of Arithmetic. Prime encoding of rhythms. Drum-set timelines. Geometrical representation of rhythms.

\section{INTRODUCTION}

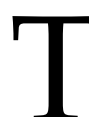
he historical blood links that connect music and mathematics are of general knowledge, dating back to about $500 \mathrm{BC}$, with Pythagoras' formulations on acoustic proportions, basically associating numbers and sounds. This represents the very foundation of what would be known as the Musica Speculativa tradition, to be pursued along the subsequent 1000 years by great

${ }^{*}$ We are very grateful to the anonymous reviewers of our article, who, with their suggestions, helped us to improve it. Received: September 27th, 2021 Approved: November 12th, 2021 
mathematicians/physicists like Euclid, Ptolemy, Galileo, Kepler, Descartes, Newton, and Euler, involving a vast range of aspects, strongly concentrated on pitch relations.

This noble tradition was reborn in a way during the twentieth century, with Schoenberg's twelve-tone method, which can be considered as a starting point and source of inspiration for a number of mathematical-musical formalizations that have changed and influenced profoundly the thought about musical creation. Among these, we can mention three decisive landmarks: (1) the Pitch-Set Class Theory, consolidated by the efforts of Milton Babbitt, Allen Forte, John Rahn, Jack Boss, and Joseph Straus; (2) David Lewin's Transformational Theory, which gave rise to the (3) Neo-Riemannian Theory, consolidated through collaborative and complementary approaches by Bryan Hyer, Richard Cohn, Jack Douthett and Peter Steinbach, Clifton Callender, Adrian Child, Edward Gollin, David Clampitt, Henry Klumpenhouwer, Robert Peck, David Kopp, among others. Since the advent of this true "cambrian explosion" in the realm of music theory, the interest on the use of mathematical models for formalizing compositional and analytical studies, branching or not from these three basic axes, has increased a lot among scholars around the world, evidenced by the great number of published dissertations, articles, and books on correlated topics in the last years.

In spite of the fact pitch subjects are certainly at the very center of gravity of such formulations (ultimately reflecting the focus of the three landmarks), an increase interest in respect to rhythm corresponds to a very recent and promising research trend. Such an avenue was opened especially by the implications that arise from isomorphic relations between pitch and rhythm realms. This is epitomized in a well-known article written by Justin London [15], who discusses the isomorphic relations that connect pitch and temporal domains based on a graph-theory perspective. The central element of London's proposal is the concept of Zeitnetz (associated with Cohn's idea of ski-hill graphs), as an analogous metric counterpart of the neo-Riemannian Tonnetz.

Certainly, meter has been being the most explored aspect of time in music, aiming at mathematical-like formalization. Richard Cohn [3] addresses Harald Kreb's concept of metric dissonance (extending it also to hypermetric levels) in an analysis of the Menuetto of Mozart's G-minor symphony. He uses some of his own reflections arisen in a previous study [2] as a base for a formal approach, searching for a new theory constructed from properties shared by pitch and time. Cohn [4] expands his metric theory by addressing the notion of complex hemiolas (i.e., involving the superimposition of two or more rhythmic layers conflicting by double and triple metric organizations). Aiming at a formal analytical treatment for the issue, Cohn idealizes an algebraic description of rhythms as products of powers of the two first prime numbers, 2 and 3, capturing the very essence of hemiolas, a strategy which in a sense keeps some relation to that proposed in this article. A graphic representation of conflicting layers in action in complex hemiolas (called sky-hill graph) is also provided by Cohn, as a helpful analytical tool. In a PhD dissertation, Stephen Guerra [10] adopts a theoretical framework mostly based on Cohn's formulations on metrical hierarchical levels to explore the music of Brazilian guitarist Baden Powell. In this work, Guerra proposes a model destined to address the concept of extended hemiolas, which extrapolates the conventional duple/triple rhythmic opposition. In a recent article, Guerra [11] uses his previous study as basis for another theoretical investigation motivated by the analytical observation of Powell's music, considering the manners with which six distinct samba timelines can be related by transformational classes of operations (labeled as augmentation and rotation). Meter is also one of the parameters (aside phrase structure, contrapuntal structure, pitch spelling, harmony, and key) selected by David Temperley [18] for the elaboration of a computational model focused on the cognition of musical structures, based on a system of preference rules. Mark Gotham [7] proposes to identify the principles that govern the notion of attractor tempos, responsible for the optimization of the salience of certain metric structures. For this purpose, Gotham elaborates a 
mathematical model capable of quantifying pulse salience, extended then to establish metrical salience values, according to hierarchical levels considered. The author provides an exhaustive list of metric structures based on consecutive levels of 2 and 3 groupings (p. 32), including the respective attractor indices.

Evidently, meter is not the only subject of scholarship interest with respect to musical time studied in mathematical terms. There is considerable literature that addresses specifically rhythmic issues. Catherine Guastavino and colleagues [9] propose an empirical-based investigation on flamenco rhythms directed to measurement of rhythmic similarity. The authors compare two mathematical methods for measuring similarity, namely chronotonic distance and the directed swap distance, the latter considered as the most efficient in the conclusion of the article. A distinctive trait of this approach is the use of phylogenetic analysis for depicting similarity relations between the rhythmic varieties. Francisco Gómez-Martín, Perouz Taslakian, and Godfried Toussaint [8] address two rhythmic categories, namely interlocking and Euclidean rhythms. While the latter category corresponds to "rhythms where the onsets are distributed among the pulses as evenly as possible" (p.18, italics in the original), the notion of interlocking rhythms refers to interaction of two or more rhythmic lines resulting into complex textures (as in African-Cuban music or in a heavy-metal groove), an aspect to be explored in the present study. In this sense, complementary interlocking rhythms, that form one of the possible types listed, are here of special interest. A recent empirical study by Florian Hoesl and Olivier Senn [14] is focused on the measurement of syncopation in drum-set grooves. It consists of a listening experiment, in which pairs of drums patterns, combined from a set of six popular grooves, were presented to 25 participants, who ranked them according to the degree of syncopation. The findings allow the authors to elaborate a formal model able to quantify syncopation in popular-music genres. Beyond the focus on the same instrument, there are other interesting shared characteristics by Hoesl's and Senn's article and the present study, especially associated with mathematical solutions for depicting rhythmic information. This will be commented in due time. Jay Hardesty [12] investigates the issue of similarity between rhythmic variants, generated from a collection of basic units. An original aspect of his work is the mapping of rhythmic derivations on Pascal's triangle, intended to provide a precise measurement of similarity between variants. In a recent $\mathrm{PhD}$ dissertation, Julio Herrlein [13] proposes the idea of a rhythmic set theory, in analogy to pitch-class sets, which includes a modulo-12 classification. Cohn [5] introduces the concept of funky rhythms, referred to the superimposition of triple-generated rhythmic patterns to duple-meter cycles formed by $8,16,32$, and 64 units. Cohn's model is deeply associated with Plato's ideas about acoustic relations. The author elects for initiating his exposition the so-called tresillo, ${ }_{1}^{1}$ present in several musical contexts around the world, and which represents perfectly the 3-over-2 duality in question. This model is adopted as basis in a study by Scott Murphy [16], whose main focus lies on the presence of funky rhythms in film music. As a theoretical framework for his analyses, Murphy proposes a detailed typology and catalogue 104 successions of 2s an/or 3s (durations, taking the sixteenth note as unit) with cardinalities from two to six elements, organizing them inside an Euler diagram, according to five categories by him established: the binary oppositions "maximally/minimally even" and "trochaic" /"iambic", and the unary "platonic".

The present article proposes a new and original manner to address rhythmic patterns, by associating their respective configurations of onsets with particular codes. This is achieved

\footnotetext{
${ }^{1}$ Tresillo is how is mostly known a rhythmic cell formed by the asymmetric division $<3,3,2>$ of an eight-pulse temporal span. Normally associated with Cuban music (commonly as the "tresillo clave"), this pattern is also present in a wide geographical spectrum, especially in Sub-Sahara musical cultures and Latin-America popular-music genres (baião, milonga, habanera, rumba, cha-cha, tango, maxixe, guajira, etc.).
} 
through some principles and formulations of Number Theory, notably the Fundamental Theorem of Arithmetic and a new function inspired by the function known as Gödel numbering. Aiming at obtaining concrete practical results and immediate applications of what will be discussed in theoretical terms along this study we direct our focus initially on the particular universe of drum-set grooves. As it will be demonstrated, the strategy of encoding rhythms (either individually or superimposed) as integers have multiple advantages, considering both analytical as compositional perspectives, opening some possible new avenues for further exploration. Before properly describing the mathematical framework that grounds this proposal, it is necessary to present the main conceptual and terminological references with respect to musical constructs adopted in our approach. These are derived from the studies of mathematical properties of rhythm as discussed by Toussaint in his 2013 book, The Geometry of Musical Rhythm [19].

\section{TOUSSAINT'S GEOMETRICAL APPROACH OF RHYTHM}

In his book, Toussaint proposes a very original manner to address rhythmic and metrical organizations. Adopting essentially an approach which involves several mathematical domains, but focusing especially on geometrical representation, Toussaint aims to systematically investigate the reasons why some rhythms are more preferable (or "good", on his terms) than others regardless of musical style, genre, epoch, or cultural context - simply by exploring some inherent mathematical properties that these rhythms have.

Four concepts of paramount importance for the present study, namely the notions of pulse, onset, inter-onset intervals, and timeline, are defined in separated passages of Toussaint's introductory notes:

The term "pulse" is used in this book to denote the location at which a sound or attack may be realized [19, p. 5].

The starting and ending times of the notes are the onsets and offsets, respectively. In the case of pure rhythms consisting of beats (attacks), it is assumed that there are no sustained notes, and thus we dispense with the offsets altogether. In this setting, the inter-onset-duration intervals are simply the durations between two consecutive attacks [19, p. 10].

In much traditional, classical, and contemporary music around the world, one hears a distinctive and characteristic rhythm that appears to be an essential feature of the music, that stands out above the other rhythms, and that repeats throughout most if not the entire piece. Sometimes this essential feature will be merely an isochronous pulsation without any recognizable periodicity. At other times, the music will be characterized by unique periodic patterns. These special rhythms are generally called timelines. Timelines should be distinguished from the more general term rhythmic ostinatos [19, p. 13].

Figure 1 tries to combine these concepts in a unique simple illustration, using musical notation. Observe that the five onsets of the rhythm (or timeline) are distributed inside a time-span of eight pulses, considering the eighth note - the shorter value in this context - as the unit of measurement.

Among 4,368 possible five-onset-in-sixteen-pulse timelines, Toussaint distinguishes six special "good rhythms", identified by him as Shiko, Son, Rumba, Soukous, Gahu, and Bossa-nova. A

\footnotetext{
${ }^{2}$ Toussaint informs that the names adopted "reflect the terminology perhaps most well known in the Western popular
} 


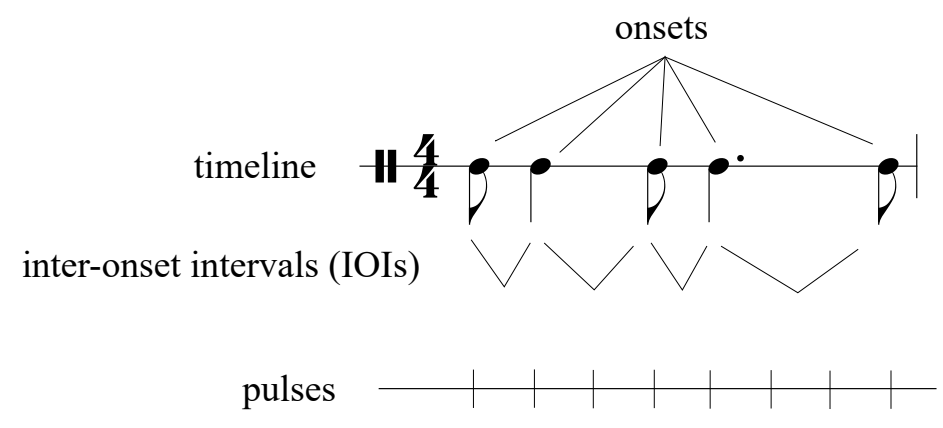

Figure 1: Example of timeline, pulses, onsets, and inter-onset intervals.

number of mathematical properties of these rhythms are discussed along the chapters, allowing for Toussaint to provide systematical comparisons. In order to properly studying these (and many other) rhythms, Toussaint presents several notation types, adopting some of them as preferential. Figure 2 illustrates the use of four of these types (binary, box, interval-vector, and polygonal) for describing the rhythm of Figure 1.

The two first types are positional descriptions of rhythms, that is, they indicate presence (1s in the former, dots in the latter) or absence (0s, empty spaces) of onsets in a given universe of pulses. On the other hand, interval-vector notation informs durational distances (measured as multiples of the considered unit) between onsets. Polygonal notation combines the two basic categories-positional and intervallic-in a geometrical representation. It will be preferentially employed in the present article, due to its special advantages and clarity, eventually complemented by the information conveyed by IOI vectors. ${ }^{3}$ Pulses are represented as little gray-line circles that divide the large circle. They are numbered from zero to $n-1$, where $n$ is the number of pulses the timeline is associated with. Onsets form vertices of a polygon (that ultimately represents the rhythm in question). The sizes of the polygon's edges are proportional to the IOIs, with their magnitude corresponding to the number of segments in the circle that they cover (for example, the edge connecting pulses 4 and 7 covers three segments, namely 4-5, 5-6, and 5-7, corresponding therefore to a IOI of three unities). From now on, let us call this notation a necklace representation, adopting Toussaint's terminology. Given the adopted set of basic concepts, terms, and rhythmic representations, we can go on with the mathematical framework which grounds the proposal.

\section{Rhythmic ENCODing}

In his article of 1931 entitled "On Formally Undecidable Propositions of Principia Mathematica and Related Systems", Austro-Hungarian mathematician Kurt Gödel (1906-1978) proved the First Incompleteness Theorem. As presented in the Britannica Encyclopaedia homepage : "[The theorem] states that any integer greater than 1 can be expressed as the product of prime numbers in only one way." It is of central importance to add that Gödel numberings are used for expressing any sequence of symbols, as mathematical formulas and even formal proofs of theorems.

The proof of this theorem has three well-defined parts. In the first one, Gödel proposes an innovative strategy, by associating each symbol/formula considered in the formal system F with

media, and I use them here purely for convenience rather than the establishment of any historical priority or cultural entitlement" [19, p. 28].

${ }^{3}$ Polygonal representation is also preferential for Toussaint, which is especially associated with the possibility of evidencing symmetrical relations of several types, an omnipresent and very important topic in his book.

${ }^{4}$ https:/ / www.britannica.com/topic/Godels-first-incompleteness-theorem 


\section{REPRESENTATIONS}

musical

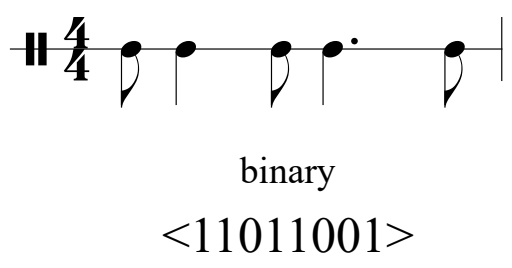

box notation

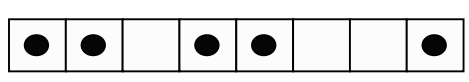

interval-vector

$<12131>$

as a polygon (necklace)

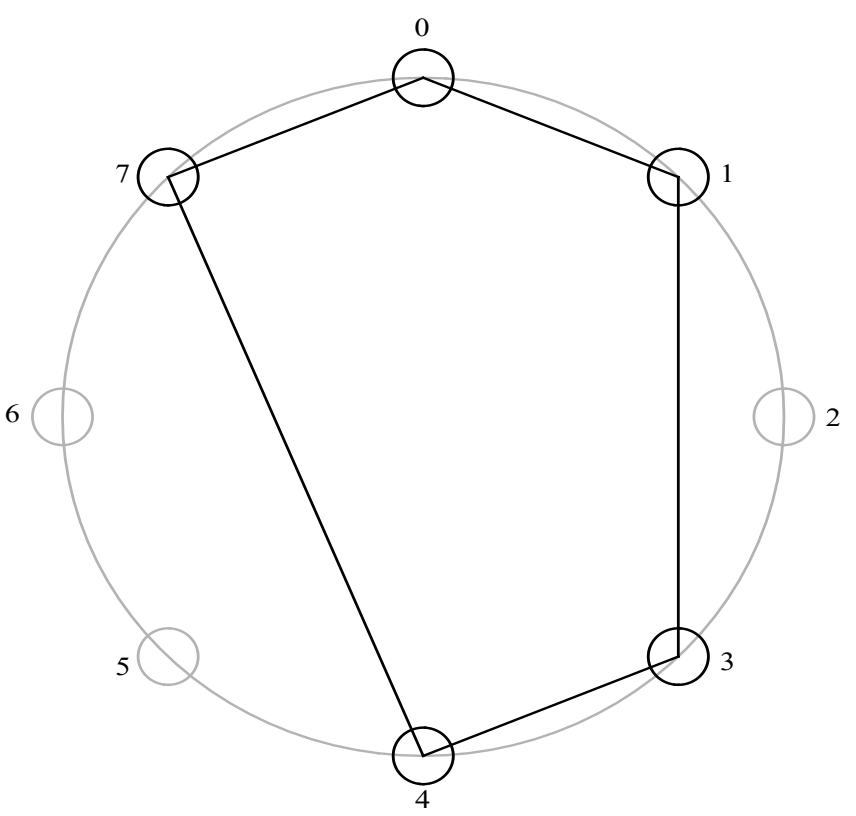

Figure 2: Four equivalent representations for the example of Figure 1. 


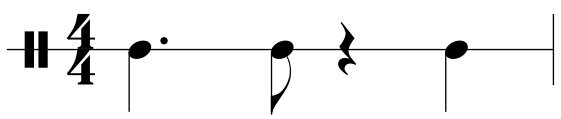

Figure 3: A simple rhythmic motive with three onsets.

an integer (called Gödel number), so that such symbol/formula is only retrievable from that number. ${ }^{5}$ The strategy adopted by Gödel inspires similar intentions in this article, more specifically with respect to the creation of a unique association between formal musical representations (more specifically, rhythmic) and codes expressed as integers, in order to allow the recovery of the former from the latter. ${ }^{6}$ In fact, more than just encoding numerically the rhythms, we are also interested in relating mapping and overlapping of musical streams through arithmetic operations involving their generating codes.

Keeping in the focus this central goal, it is paramount at this moment to recall the main result of the integer arithmetic, the Fundamental Theorem of Arithmetic (FTA, for short). This result was first proved by German mathematician Carl Friedrich Gauss (1777-1855) at the very end of the eighteenth century and published in 1801 on his treatise Disquisitiones Arithmeticae. The proposition 30, 31, and 32 of the book VII of Euclid's Elements presents results that show how close the Greeks were to the FTA, 2000 years before Gauss.

The Fundamental Theorem of Arithmetic: Let $\mathrm{N}$ be an integer other than 0,1 , and -1 . Then there exist positive prime numbers $\mathrm{p}_{1}<\mathrm{p}_{2}<\ldots<\mathrm{p}_{\mathrm{k}}$ and positive integers $\mathrm{n}_{1}, \mathrm{n}_{2}, \ldots, \mathrm{n}_{\mathrm{k}}$ such that

$$
N= \pm p_{1}^{n_{1}} \times p_{2}^{n_{2}} \times \ldots \times p_{k}^{n_{k}}
$$

Disregarding permutations of the factors, this decomposition is unique. If we extend the perception of such a result beyond the specificities involved, we will realize that, more than a single decomposition of an integer as a product of prime-number powers, FTA explains the possibility of encoding a vector containing k numeric information $\left\langle\mathrm{n}_{1}, \mathrm{n}_{2}, \ldots, \mathrm{n}_{\mathrm{k}}\right\rangle$ as a single number, in a bijective way. For example, the decomposition presented by equality $360=2^{3} \times 3^{2}$ $\times 5^{1}$ can be understood as the encoding of the information conveyed by the vector $\langle 3,2,1\rangle$, formed by the exponents of the prime product, in the number 360 . In this sense, the involved prime numbers address the number of coordinates/information conveyed by the vector, while its exponents constitute the information itself. In the present article, musical representations will adopt this same encoding process.

As a very simple example, consider the following rhythmic motive, corresponding to a possible manifestation of the tresillo clave (Figure 3).

Considering the duration of an eighth note as a unit of informational space, this representation configures Toussaint's binary vector $<10010010>$. If we use FTA for encoding this vector, we have this unique possible factoring:

$$
2^{1} \times 3^{0} \times 5^{0} \times 7^{1} \times 11^{0} \times 13^{0} \times 17^{1} \times 19^{0}=238
$$

\footnotetext{
${ }^{5}$ For a detailed presentation of this algorithm, which came to be known as Gödel numbering, as well as a comprehensive explanation of Gödel's achievements, see [17].

${ }^{6}$ As far goes our knowledge, the idea of expressing rhythmic formulas through the use of the Gödel-numbering algorithm was not yet explored in academic studies. Considering other aspects of musical construction, see the strategy proposed by [1] for codifying as univocal integers strings of genealogical descriptions of variations algorithmically produced. In this case, the order number of a variant located in the context of a given sequence of previous generation is represented as a series of exponents raising a sequence of prime bases, whose product corresponds to an integer that stands for the precise and formal identification of the variation in question.
} 
Therefore, the number 238 uniquely identifies the rhythmic motive of Figure 3. In other terms, 238 is a numeric code univocally related to this specific musical representation.

A fundamental point to be observed is that the product of the codes referring to two distinct musical representations (since both share the same durational unit) will be the code related to the representation of the superimposition of those two representations, if they start at the same instant and have the same time-span pattern. Consider, for example, the distribution of the onsets of the tresillo of Figure 3 in two streams, as if played by two distinct instruments (Figure 4).
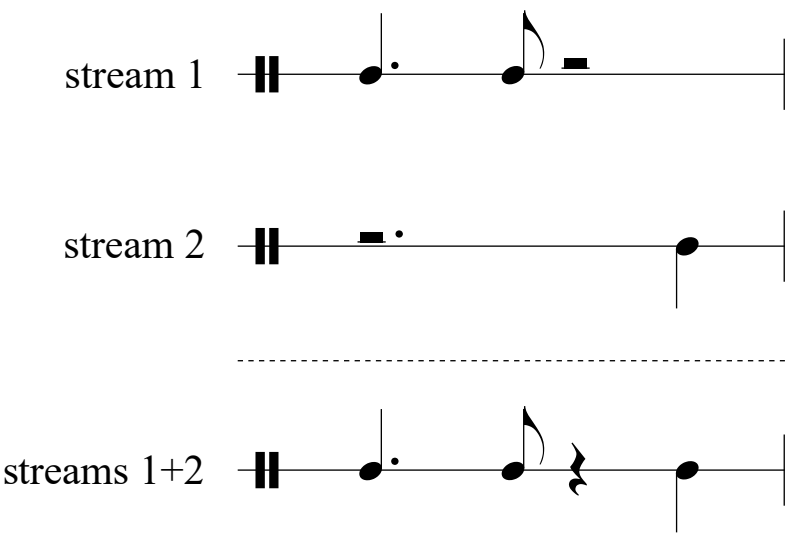

Figure 4: Distribution of the onsets of the rhythm of Figure 3 in two separated streams.

Thus, we have:

$2^{1} \times 3^{0} \times 5^{0} \times 7^{1} \times 11^{0} \times 13^{0} \times 17^{0} \times 19^{0}=14$ (code of the stream 1$)$

$2^{0} \times 3^{0} \times 5^{0} \times 7^{0} \times 11^{0} \times 13^{0} \times 17^{1} \times 19^{0}=17($ code of the stream 2$)$

In this case, the product of the two codes, $17 \times 14=238$, equals the representation code referring to superimposed rhythms, as shown in Figure 3. Thus, we can affirm that the greatest common divisor ( $\mathrm{gcd}$ ) of the codes related to $\mathrm{N}$ representations in a same informational context will be the representation code referring to the commonly shared information. In the absence of any shared information, gcd equals 1 , which is the code for the representation associated with the null vector.

For specific purposes, let us define an informational unit (IU, for short) as the smallest durational value considered in a given rhythmic context, which is equivalent to Toussaint's notion of pulse, as presented in the previous section. The strategy of setting IUs as contextual-dependent instead of adopting a fixed unit (say, the 16th note) is motivated by an intention of minimizing computational cost. Figure 5 illustrates the contextual nature of IUs with different situations. ${ }^{7}$ In two distinct depictions of the tresillo (labeled as a and b), the informational unit corresponds to the 8th note, disregarding if it is explicitly or not present in the rhythm. In (c) the smallest value is clearly the 16 th note. The last rhythm, however, combines groups of 8th notes (figure that divides the beat by 2 ) and of triplets (a ternary division). Since the least common multiple referred to these two values ( 2 and 3 ) is 6, this means that the implicit sixteenth triplet must be assigned as IU in the case.

In addition, it is also possible to incorporate other types of information in a code as, dynamics, for example. This is reflected in the algorithm by defining other possible values (beyond 0 and 1 ) to be assumed by the exponents. We can, for example, establish three possible dynamic levels for

\footnotetext{
${ }^{7}$ Observe how in each rhythm the pulses are counted from 0 to $n-1$, where $n$ is the number of IUs present in the rhythm.
} 
(a)

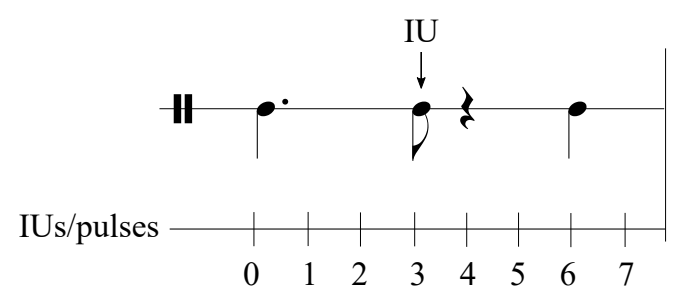

(b)

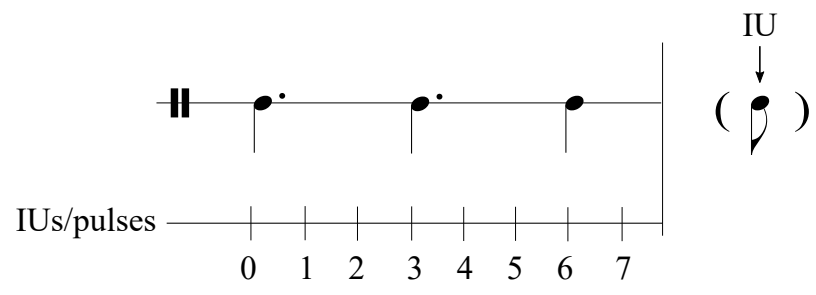

(c)

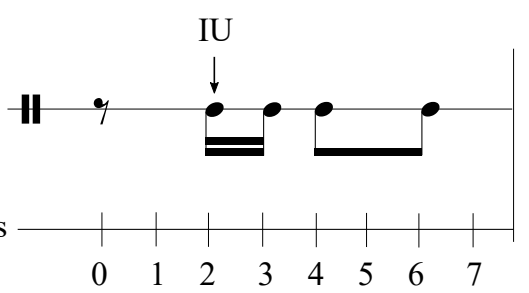

(d)

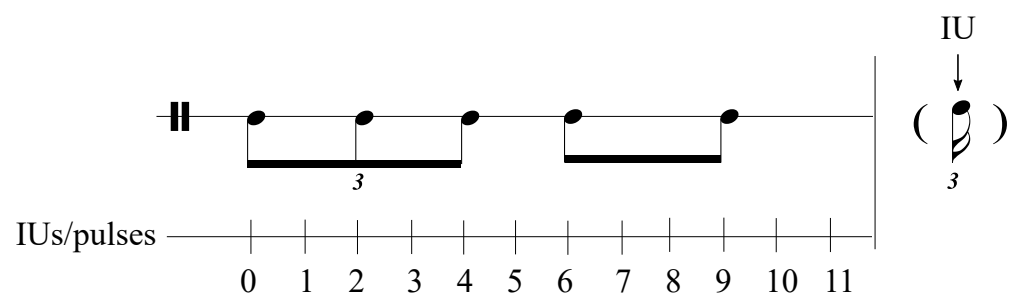

Figure 5: Four rhythms with different IUs.

any onset of a given rhythm: 1 (default or "flat"), 2 ("ghost" note) or 3 (accented). ${ }^{8}$ In this sense, the intention of adding a phenomenal accent to the third onset of the tresillo of Figure 3 would result in a new code: $2^{1} \times 3^{0} \times 5^{0} \times 7^{1} \times 11^{0} \times 13^{0} \times 17^{3} \times 19^{0}=68782$. Therefore, with each unit mapped by a prime number, it is possible to assign different sets of informational values, depending on the level of detail that one intends. Evidently, the more information is included, the bigger the integers that represent the codes. Anyway, and this is of paramount importance, there will be always a one-to-one correspondence between a code and a rhythmic context which it is intended to represent.

We propose the following formalization of the code, associated with a measurable rhythmic representation by a determined unit (IU), retrievable with $\mathrm{k}$ information.

Definition: Let $R$ be a rhythmic representation measurable by an informational unit $U$, with

\footnotetext{
${ }^{8}$ These numbers were arbitrarily chosen. As it will be discussed in due time, the same strategy can be applied to other parameters, like timbral identification, for example.
} 
measure $k \cdot{ }^{9}$ Let $I_{\mathrm{n}} \subset \mathbb{Z}_{+}^{*}, 1 \leq n \leq k$ be the sets of informational values of the coordinates of the vector $\left(\mathrm{c}_{1}, \mathrm{c}_{2}, \ldots, \mathrm{c}_{\mathrm{k}}\right)$, associated with the representation $R$.

Let $B=\left\{p_{1}, p_{2}, \ldots, p_{k}\right\}$ a set made up of ordered, distinct, and positive prime numbers $\left(\mathrm{p}_{1}=\right.$ 2 ). The code $M$ of the representation $R$, in relation to the set $B$, is given by:

$$
M=p_{1}^{c_{1}} \times p_{2}^{c_{2}} \ldots p_{k-1}^{c_{\mathrm{k}-1}} \times p_{k}^{c_{\mathrm{k}}}=\prod_{n=1}^{k} p_{n}^{c_{\mathrm{n}}}
$$

The $\mathrm{M}$ code is unique and retrieves all information contained in the information sets associated with a given rhythmic representation.

As an illustration of the type of application that will be considered in this study, consider a simple eight-pulse drums pattern composed by the superimposition of three rhythmic lines, played by the hi-hat (stream 1), snare drum (stream 2), and bass drum (stream 3), as depicted in Figure 6.

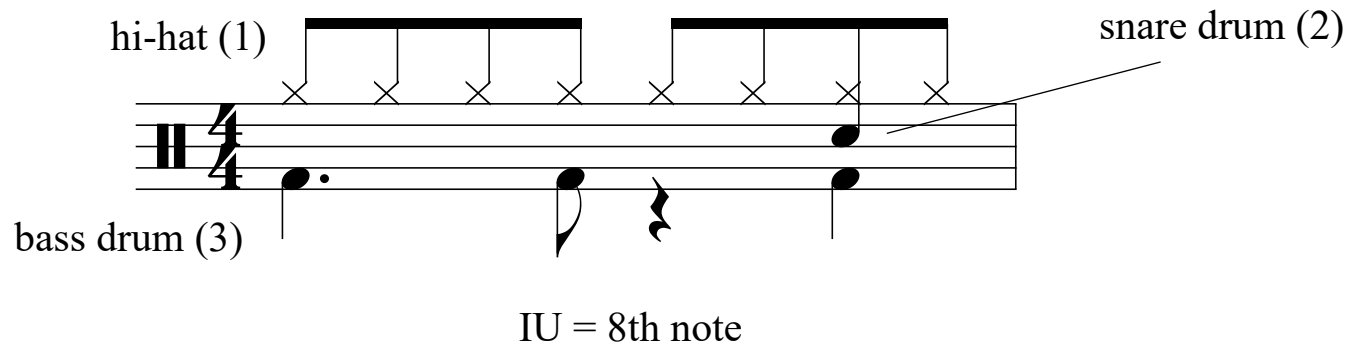

Figure 6: Example of a simple three-stream drums pattern.

The respective codes are calculated as following:

$$
\begin{gathered}
M_{\text {hi-hat }}=2^{1} \times 3^{1} \times 5^{1} \times 7^{1} \times 11^{1} \times 13^{1} \times 17^{1} \times 19^{1}=9699690 \\
M_{\text {snare }}=2^{0} \times 3^{0} \times 5^{0} \times 7^{0} \times 11^{0} \times 13^{0} \times 17^{1} \times 19^{0}=17 \\
M_{\text {bass }}=2^{1} \times 3^{0} \times 5^{0} \times 7^{1} \times 11^{0} \times 13^{0} \times 17^{1} \times 19^{0}=238
\end{gathered}
$$

It should be noted that $\operatorname{gcd}(17,238,9699690)=17$, which is precisely the prime number associated with the seventh pulse, the unique point where onsets of the three drum pieces coincide. In this sense, the concept of greater common divisor and the disposition of the rhythmic attacks in the pattern are closely related. ${ }^{10}$

\section{Algorithmic implementation}

The combination of the ideas of the Gödel-numbering algorithm with vector/necklace representation provides the necessary means for the elaboration of a robust theoretical-methodological apparatus addressing rhythm, with both analytical and compositional applications. As suggested in the previous section, this strategy allows for encoding any possible rhythmic configuration (or even superimposed rhythmic lines, as happens in drum-set grooves) as a unique integer (or a tuple of integers, as it will be discussed), which ultimately will constitute a precise identifier of such rhythm. Put another way, given a rhythm $\mathrm{R}$, expressed in one of the notational alternatives proposed by Toussaint (we will adopt in this study vector and necklace types), there is a particular

\footnotetext{
${ }^{9}$ Depending on the musical context in question, any durational value can be considered as rhythmic unit. By default, we propose the sixteenth note for this role.

${ }^{10}$ It is important to add that this model for drum-groove encoding is a simple, initial version that to be be replaced by a more complex one, whose description is presented along the next section.
} 
integer M (standing for "M... code") ${ }^{11}$ that will represent univocally R. Conversely, considering a given integer as $\mathrm{M}$, playing backwards the same process, we can obtain algebraic and geometric representations of the related rhythm $R$. The present section describes the algorithms and processes employed for the accomplishment of both tasks. In addition, practical illustration of their potential application in analysis and composition will be provided.

\section{i. Isolated rhythms}

Aiming at a more clear and gradual explanation of the structure of the algorithms, let us start with a simple rhythm played by a single drum-kit piece, say, the snare. As shown in Figure 7, the timeline in question consists of four onsets in a groove of sixteenth pulses. In this context, the eighth note is the lesser durational value and, therefore, it is the IU to be considered along the process.
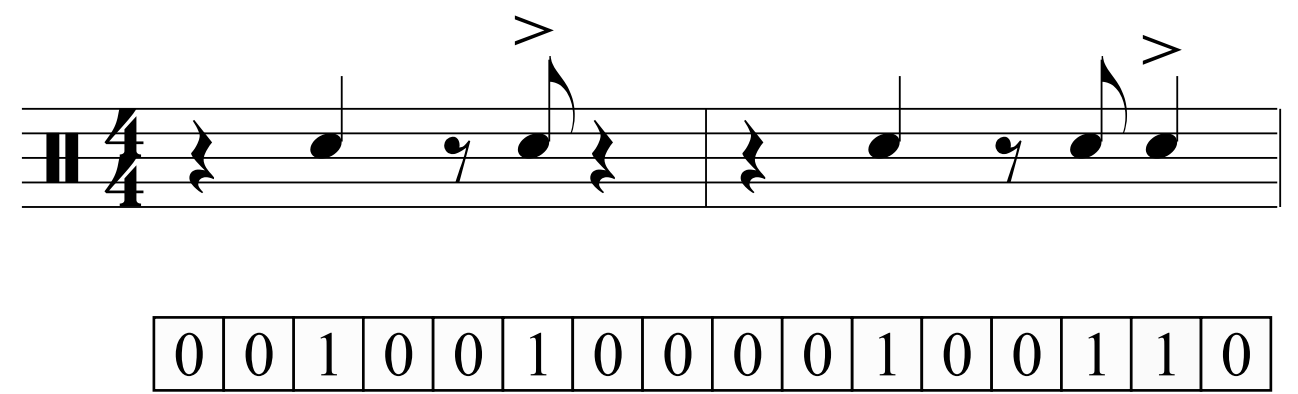

Figure 7: Example of an isolated rhythm written for snare drums, in musical and binary notation.

Firstly, the rhythm must be transcribed from musical to binary (positional) notation, with 1s corresponding to onset points, as shown beneath the score. The conventions adopted with respect to dynamic levels (introduced in the previous section) are incorporated into the positional vector, according to the following instructions: (a) replace 1 by 2 in the case of a ghost-note onset; (b) replace 1 by 3 in the case of an accented onset (and do nothing in normal onsets). Thus, there are two substitutions in our example (Figure 8).

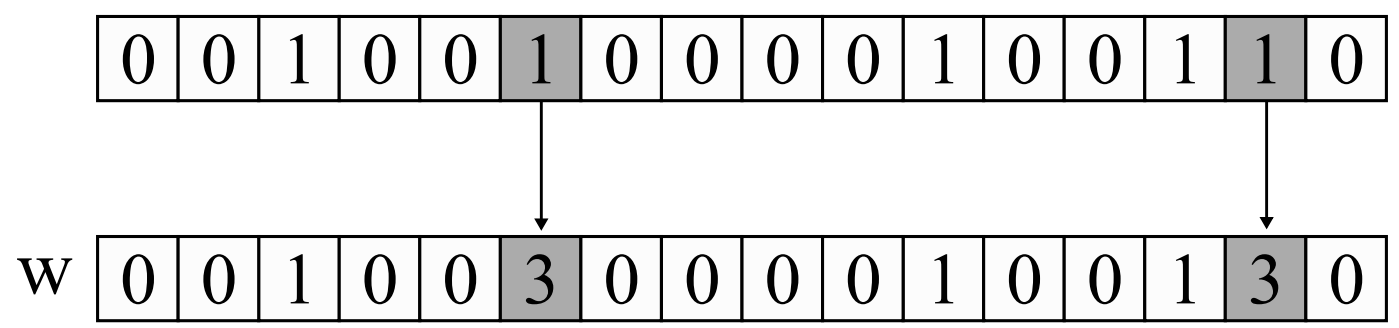

Figure 8: Formation of vector w, using as example the rhythm of Figure 7.

Let us refer to this latter structure as the weighted vector $\mathrm{w}$, which ultimately represents a given rhythm $\mathrm{R}$ with respect to its onset points and micro-dynamic fluctuations. Vector $\mathrm{w}$ is then inputted in the program PlotRhythm, ${ }^{12}$ which produces the necklace representation of $\mathrm{R}$ as

\footnotetext{
${ }^{11}$ This designation refers to Dr. Carlos Mathias, the researcher who idealized the code, and one of the co-authors of this article.

${ }^{12}$ This and the all programs which will be mentioned in this article were implemented in Matlab language.
} 
exemplified in Figure 9 (thick-line nodes correspond to the accented onsets). Nodes of normal width refer to normal onsets. Ghost-note onsets would be represented by dashed-line nodes.

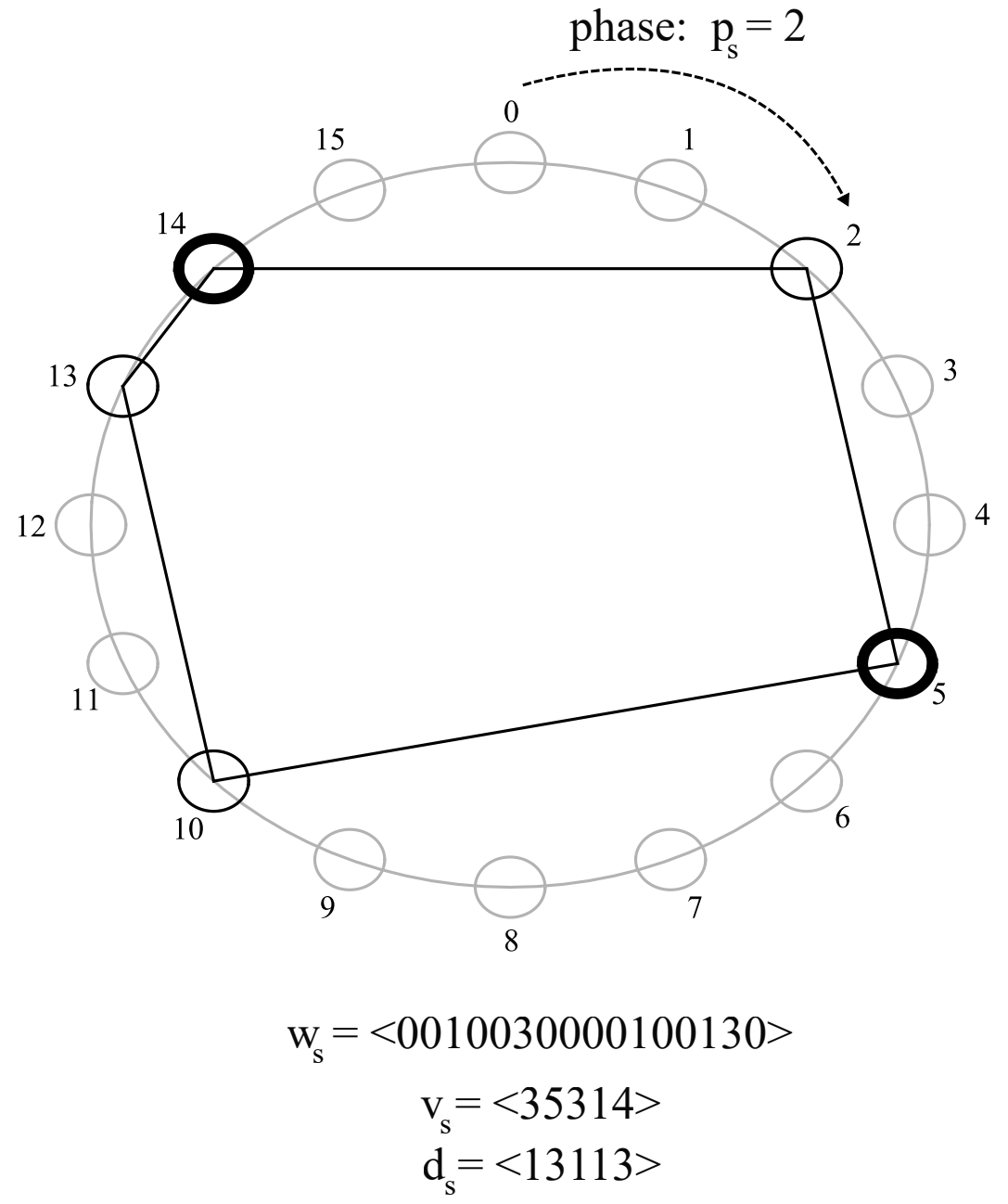

Figure 9: Necklace representation of the rhythm of Figure 7.

Before continuing with the encoding of $\mathrm{R}$, there are some new concepts and conventions to be introduced. Define the phase (denoted by p) of a rhythm as the distance (measured in IUs) between the starting point of the cycle/timeline (always indicated by the zero "hour" in the necklace) and the position of the first onset. In the case of our example, the rhythm has phase $\mathrm{p}_{\mathrm{s}}=$ 2. The intervallic vector $\mathrm{v}$ proposes a more compact representation through the depiction of the sequence of differences (measured in IUs or pulses) between the onsets. ${ }^{13}$ In the present case, $\mathrm{v}_{\mathrm{s}}=$ $<35314>$. ${ }^{14}$ Define also the dynamic vector $\mathrm{d}$ as the sequence of dynamic levels in a one-to-one relation with the onsets of $\mathrm{R}\left(\mathrm{d}_{\mathrm{s}}=<13113>\right.$ in the exemplified case).

The algorithm that encodes $\mathrm{R}$, called TranslateRhythm, uses as input vectors $\mathrm{w}, \mathrm{v}, \mathrm{d}$, and phase $\mathrm{p}$. It functions basically as follows: Firstly, it forms a sequence of prime bases $\mathrm{P}$, whose length equals

\footnotetext{
${ }^{13}$ In other words, $\mathrm{v}$ denotes a sequence of IOIs of R.

${ }^{14}$ Observe that these differences are proportional to the lengths of the edges in the polygon of Figure 9. Notice also that it is necessary to include in $\mathrm{v}$ the interval between the last and the initial onsets of the rhythm (4 pulses in the example), closing the circuit, which is perfectly consistent with the cyclic nature of a timeline.
} 
the cardinality of the timeline. Considering that, in our example, the snare pattern has 16 pulses, then $\mathrm{P}=\langle 2,3,5,7,11,13,17,19,23,29,31,37,41,43,47,53\rangle$. After this, the members of $\mathrm{P}$ that correspond to non-zero entries of $\mathrm{w}$ are copied into another vector, $\mathrm{P}_{\mathrm{R}}$, as shown in Figure 10.

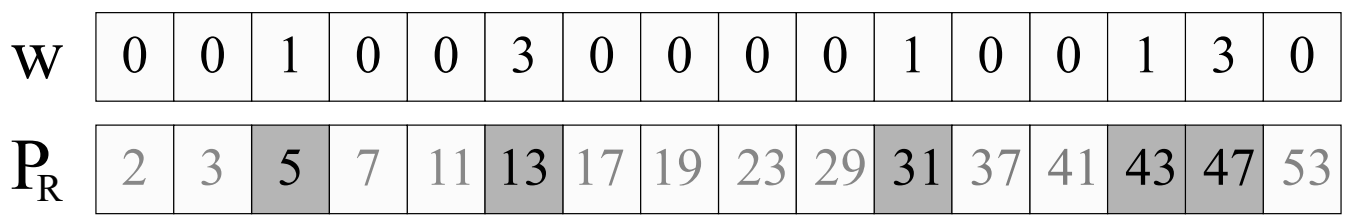

Figure 10: Formation of vector $P_{R}$, using as example the rhythm of Figure 7.

The integer resulting from the product of these prime numbers is the code referred to $R$. Let us label this integer as $G_{R}$. Considering the snare rhythm, we have $G_{R_{S}}=5 \times 13 \times 31 \times 43 \times 47=$ 4072315.

Let now $G_{D}$ be the integer that encodes the dynamic pattern of $R$. With the goal of minimizing the magnitude of this number, a different strategy is used in this case. Since there is a one-to-one mapping between the dynamic indications of vector $\mathrm{d}$ and the respective onsets of vector $\mathrm{v}$, it is not necessary to locate their positions in the cycle (as it was done with the onsets). Therefore, it is enough to associate the $n$ elements of $d\left(d_{1}, d_{2}, \ldots, d_{n}\right)$ as exponents of the first $n$ prime numbers $\left(p_{1}, p_{2}, \ldots, p_{n}\right)$. Thus, $G_{D}$ will be the product of these powered bases. For the snare timeline, we have: $\mathrm{G}_{\mathrm{DS}_{\mathrm{S}}}=2^{1} \times 3^{3} \times 51 \times 7^{1} \times 11^{3}=2515590$.

Finally, the code $M$ of $R$, is represented by the triplet $M=\left(c, G_{R}, G_{D}\right)$, where $c$ is the length of the timeline (measured in IUs), $G_{R}$ is the Gödel number related to $R$, and $G_{D}$ is the Gödel number referred to the particular dynamic pattern of $R$. Thus, in our example, $M_{S}=(16,4072315,2515590)$.

The reversal process, that is, for obtaining the original rhythm from the code, is considerably simple, through factoring both integers $G_{R}$ and $G_{D}$, considering also the context (i.e., the cycle length). The decoding is operated by another algorithm, called TranslateCode, whose input is M. Taking the case of our example, the algorithm proceeds as depicted in Figure 11. However, as suggested in the sixth step of the algorithm, it is not possible to recover exactly the original musical notation, since the whole process deals with onsets (and IOIs), rather than properly durations. ${ }^{15}$ Put another way, the three possible interpretations depicted at the bottom of Figure 11, or any other that obeys the positions of the onsets in the same 16-pulse context, will be considered perfectly equivalent with the respect to onset/dynamic patterns. Actually, since we are working with drums, the determination of the durations of the attacks is entirely immaterial. Given this, from now on, we will adopt as the preferential the simplest and clearest (in the drummer's perspective) possible notation for representing both the original and recovered rhythm. The next section examines a more complex situation, involving superimposition of rhythms, as it normally occurs in common drum grooves.

\footnotetext{
${ }^{15}$ Evidently, there are some special situations in which drum-set durations matter, as in snare rolls or in the reverberation of attacks on crash or splash cymbals. For simplicity, we will not consider such situations in the present model.
} 


\begin{tabular}{|l}
\hline \multicolumn{10}{|c|}{$\mathrm{M}_{\mathrm{s}}=(16,4072315,2515590)$} \\
$1 . \mathrm{c}=16 \longrightarrow \quad 0$ \\
0
\end{tabular}

2. factor $\mathrm{G}_{\mathrm{R}} \longrightarrow 4072315=5 \times 13 \times 31 \times 43 \times 47$

$$
\begin{aligned}
& 3^{\text {rd }} \quad 6^{\text {st }} 11^{\text {st }} 14^{\text {st }} 15^{\text {st }} \\
& \text { (positions on the prime sequence) }
\end{aligned}
$$

3. enter positions in the zeroed vector (step 1) as $1 \mathrm{~s}$

$$
\begin{array}{|l|l|l|l|l|l|l|l|l|l|l|l|l|l|l|l|}
\hline 0 & 0 & 1 & 0 & 0 & 1 & 0 & 0 & 0 & 0 & 1 & 0 & 0 & 1 & 1 & 0 \\
\hline
\end{array}
$$

4. factor $\mathrm{G}_{\mathrm{D}} \longrightarrow 2515590=2^{1} \times 3^{3} \times 5^{1} \times 7^{1} \times 11^{3}$

5. take the exponents and enter them in the vector of positions (step 3). replacing non-zeroed entries in the same order, obtaining vector $\mathrm{w}$

\begin{tabular}{ll|l|l|l|l|l|l|l|l|l|l|l|l|l|l|l|}
\multicolumn{1}{c|}{} & \multicolumn{1}{c}{3} \\
$\mathrm{~W}$ & 0 & 0 & 1 & 0 & 0 & 3 & 0 & 0 & 0 & 0 & 1 & 0 & 0 & 1 & 3 & 0 \\
\hline
\end{tabular}

6. write the resulting vector in musical notation (optional)

$$
\begin{array}{|l|l|l|l|l|l|l|l|l|l|l|l|l|l|l|l|}
\hline 0 & 0 & 1 & 0 & 0 & 3 & 0 & 0 & 0 & 0 & 1 & 0 & 0 & 1 & 3 & 0 \\
\hline
\end{array}
$$

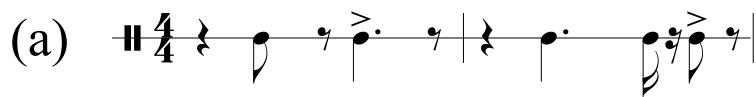

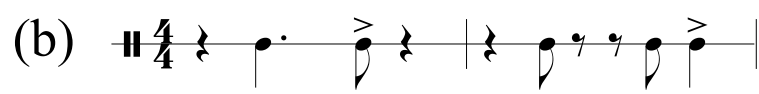

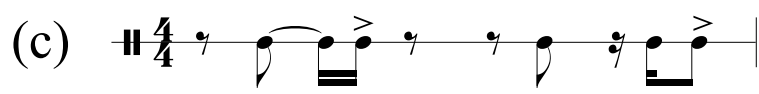

etc.

Figure 11: Basic algorithm of TranslateCode for recovering an isolated rhythm $R$ from a $M$ code. 


\section{ii. Drum grooves}

Speculating about what was suggested in the third section of this article, it is possible to consider other additional "dimensions" for the onsets of a rhythm, besides dynamics. Information concerning timbre, especially, is a very useful feature to be added with respect to drums. Provided that clear conventions are previously established by the analyst, even complex drum patterns can be encoded with this technology. Consider as an illustration of this possibility the following conventions (Table 1). They can freely combine dynamic and timbral information (as in the examples) in order to adapt to genre (heavy rock, blues, bossa, salsa, etc.) or other contextual conditions.

Table 1: Two possible convention charts for drum-set encoding (shaded cells refer to timbral features).

\begin{tabular}{ccccc}
\hline \hline streams & 1 & 1 & 3 & 4 \\
\hline \multicolumn{5}{c}{ (case a) } \\
\hline 1 & bass drum & $\begin{array}{c}\text { accented onset } \\
\text { ghost note }\end{array}$ & - & accented onset \\
2 & snare drum & rim shot \\
closed hi-hat & opened hi-hat & - & - \\
\hline \multicolumn{5}{c}{ (case b) } \\
2 & bass drum & accented onset & - & - \\
3 & snare drum & low tom & mid tom & high tom \\
closed hi-hat & opened hi-hat & ride cymbal & attack cymbal \\
\hline
\end{tabular}

Let us adopt the simpler case (a) of Table 1 for addressing the superimposition of rhythms in a three-stream groove. Figure 12 adds bass-drum and hi-hat lines to the snare pattern of the previous section, forming a rock-like simple groove. The respective positional vectors can then be accordingly written. For visual clarity, no accents were added to the bass-drum rhythm.
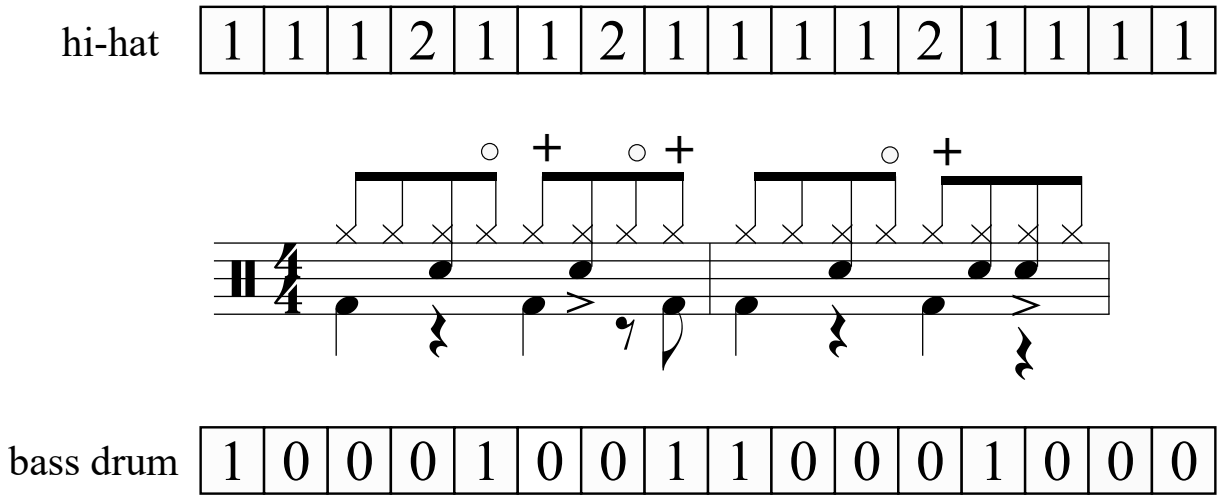

Figure 12: Drum groove obtained by superimposition of bass-drum and hi-hat rhythms to the snare pattern of Figure 7.

Since the three weight vectors $\left(\mathrm{w}_{\mathrm{b}}, \mathrm{w}_{\mathrm{s}}, \mathrm{w}_{\mathrm{h}}\right)$ share, evidently, the same cycle of 16 pulses, they can be reunited in a unique three-row matrix (denoted as T), reproducing from top to the bottom the order of the pieces in the score: ${ }^{16}$

\footnotetext{
${ }^{16}$ Hoesl and Senn [14, p. 7] also use a 3-row matrix to notate onset positions of the drum pieces. However, since they are interested in syncopation, their matrix depicts rhythmic information related only by snare and bass drums (rows 1 and 2).
} 


$$
T=\left|\begin{array}{llllllllllllllll}
1 & 1 & 1 & 2 & 1 & 1 & 2 & 1 & 1 & 1 & 1 & 2 & 1 & 1 & 1 & 1 \\
0 & 0 & 1 & 0 & 0 & 3 & 0 & 0 & 0 & 0 & 1 & 0 & 0 & 1 & 3 & 0 \\
1 & 0 & 0 & 0 & 1 & 0 & 0 & 1 & 1 & 0 & 0 & 0 & 0 & 1 & 0 & 0
\end{array}\right|
$$

Matrix $\mathrm{T}$ is then input in the program PlotRhythm, which returns the graphic representation depicted in Figure 13.

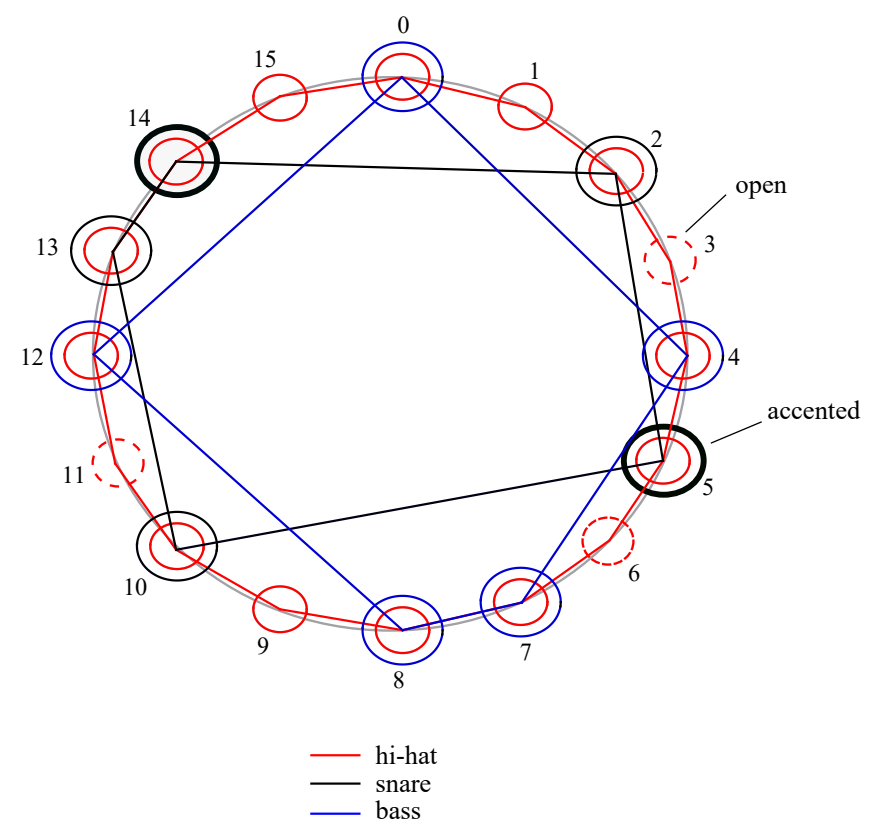

Figure 13: Necklace representation of the drum groove of Figure 12.

Due to the increase of complexity, colors are used for discriminating the pieces' rhythms. As in the simple mode, the number of edges in each polygon represents the number of onsets of the rhythms. Concentric, superimposed circles indicate coincidence of onsets involving two pieces (in the exemplified groove there are no triple coincidence). The dashed-line red nodes refer to attacks on the opened hi-hat.

The next step proceeds as it was done with the snare line. The individual weight vectors of bass drum and hi-hat are input, one at a time, in the TranslateRhythm algorithm. In the case of the bass drum, there is nothing new: the algorithm returns $M_{b}=(16,394174,1)$. Observe that $G_{D}$ $=1$, which is consistent with the fact that the bass-drum rhythm is completely flat with respect to the dynamic level. On the other hand, the calculation of $\mathrm{M}_{\mathrm{h}}$ gives rise to a problematic issue. Because its rhythm articulates all the pulses of the cycle, this implies a very huge value for GR (approximately $3.26 \times 10^{19}$ ). Apart from the awkwardness of representing an integer of such magnitude in the code, the real greatest difficult in dealing with it lies actually in the decoding process, due to the computational limitations for factoring mammoth integers like this. ${ }^{17}$

In the following, this paper will share some strategies on how to reduce the potential size of the integers involved within the structure of code $\mathrm{M}$ (for computational purposes), specifically for

The third row is reserved for what they call metric weight, associated with distinct values of syncopation, from 0 to -3 (it is important to state, as it will be clear ahead, that this meaning for "weight" is completely different from which is here adopted.

${ }^{17}$ Factoring becomes complicated with integers greater than $10^{15}$. 
the cases of rhythms that contain a lot of information. The presentation of those strategies could suggest to the reader that the intent behind this encoding technique is to generate integers able to uniquely represent a rhythm. If that were simply the case, the coordinates of the informational vectors themselves would easily fulfill that wish. The hi-hat line of Figure 12, for instance, could be naively encoded by $<1112112111121111>$. But that would only provide a source of representation that is null in terms of providing arithmetical feedbacks useful to any sort of analysis. The main concern of the encoding technique here presented is to uniquely link an arbitrary musical representation to numerical informational vectors, in such a way that the analysis of rhythmic attributes can be carried out within a robust arithmetical framework, in the sense previously described. Rhythmic superimpositions/decompositions, displacements, dynamic changes and even modulations can be easily achieved as results of the use of simple arithmetic operations such as multiplication and division. Metaphorically, it can be said that Toussaint's necklace notation is to geometry as the encoding technique here presented is to arithmetic.

The first strategy considered was to "break" $G_{R}$ into three factors, such that $G_{R}=g_{1} \times$ $\mathrm{g}_{2} \times \mathrm{g}_{3} \cdot{ }^{18}$ Although this solves perfectly the problem, it is an unsatisfactory solution, since it would force us to restructure code $\mathrm{M}$, changing it from a triple to a quintuple, in order to eventually accommodate two new entries. A quite more elegant alternative is to consider the idea of complement of a rhythm $\mathrm{R}$, denoted as $\mathrm{R}^{\mathrm{R}}$. This corresponds to the set of onsets that are not articulated by the rhythm, in the context of the cycle it is inserted in. In the case of our hi-hat example, since all pulses are articulated, its complement $\mathrm{R}^{\mathrm{R}}$ corresponds to no articulation at all, being arithmetically represented by the product of the sixteen primes, all of them powered by 0 , which equals 1. Evidently, the use of $R^{R}$ is destined not only to express, so to speak, all-pulse rhythms. As a matter of fact, a rhythm with a number of onsets greater than half of the number of pulses can be "negatively" represented by its complement.

As an illustration of this point, consider, for instance, a hypothetical hi-hat variation of the groove, with an intermittent onset pattern denoted by the weight vector $\mathrm{w}=<1011101011101101>$. Then, its complementary weight vector $\mathrm{w}_{\mathrm{c}}$ will be expressed as $\left.\mathrm{w}_{\mathrm{C}}=<0100010100010010\right\rangle$. Figure 14 provides a necklace representation of both rhythms.

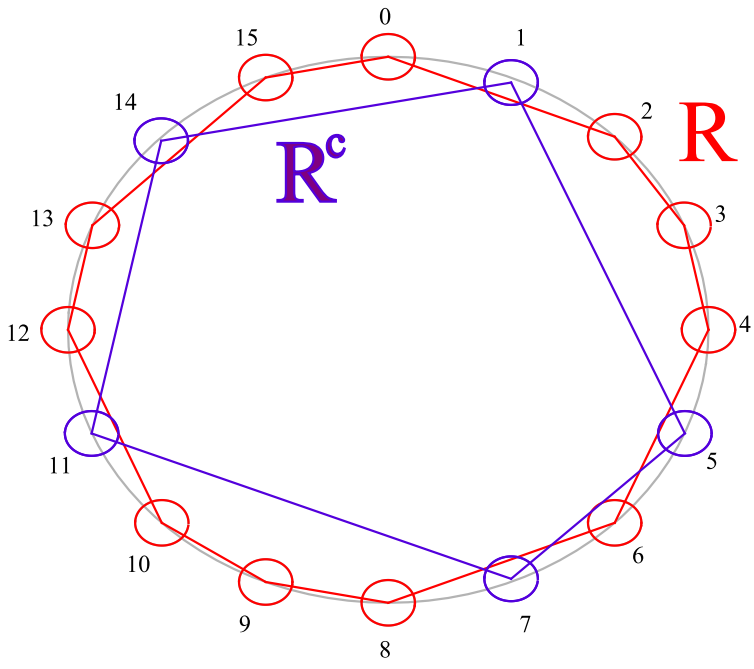

Figure 14: Necklace representation of a hypothetical rhythm $R$ and its complement $R_{c}$.

${ }^{18}$ In the present case, the values are: $g_{1}=2310 ; g_{2}=2800733$; and $g_{3}=5037203051$. 
Now, we calculate $G_{R}$ for both rhythms and compare the results. As done in ordinary cases, $\mathrm{G}_{\mathrm{R}}$ is the product of prime numbers corresponding to non-zeroed entries of the weight vector. The lowest integer calculated will then be chosen for encoding the rhythm. As shown in Table 2 the comparison of both alternatives favors the choice of the complement as the representative of the rhythm.

Table 2: Comparison between $R$ and $R_{c}$ referring to the case of Figure 14.

\begin{tabular}{cccccccccccccccccc}
\hline \hline Pulses & 0 & 1 & 2 & 3 & 4 & 5 & 6 & 7 & 8 & 9 & 10 & 11 & 12 & 13 & 14 & 15 & $\mathrm{G}_{\mathrm{R}}$ \\
Primes & 2 & 3 & 6 & 7 & 11 & 13 & 17 & 19 & 23 & 29 & 31 & 37 & 41 & 43 & 47 & 53 & \\
\hline $\mathrm{w}$ & 1 & 0 & 1 & 1 & 1 & 0 & 1 & 0 & 1 & 1 & 1 & 0 & 1 & 1 & 0 & 1 & $2.53 \times 10^{13}$ \\
\hline $\mathrm{w}^{\mathrm{c}}$ & 0 & 1 & 0 & 0 & 0 & 1 & 0 & 1 & 0 & 0 & 0 & 1 & 0 & 0 & 1 & 0 & $\mathbf{1 2 8 8 5 9 9}$ \\
\hline
\end{tabular}

The indication in the code $\mathrm{M}$ that a rhythm is represented by its complement is accomplished through a very simple artifice: the multiplication of the respective $G_{R}$ by -1 .

On the other hand, if the complement is chosen for representing a given rhythm, its $G_{D}$ shall be calculated in a different way from the ordinary case. Actually, the algorithm is very simple and can be described in two steps: (a) subtract 1 from the entries of original vector $w$, an action that we will represent by $\mathrm{w}-1$; (b) power the primes with the resulting vector (as it was done in the calculating of $\mathrm{G}_{\mathrm{R}}$ ) and calculate their product. In the case of the hypothetical example of Figure 14, we have then the subtraction $\left.\mathrm{w}^{\mathrm{c}}-1=<0000000000000000\right\rangle$, implying then $\mathrm{G}_{\mathrm{D}}=1$ (a not surprising result, since $\mathrm{w}^{\mathrm{c}}$ is perfectly flat). We can then encode this rhythm as $\mathrm{M}=(16$, $-1288599,1)$.

We can now return to the original hi-hat pattern and encode it properly. Recall that its weight vector (that includes some opened onsets) is expressed as $w=<1112112111121111>$. Since the rhythm saturates the onset space, $G_{R}=-1$. For calculating $G_{D}$, we take $w-1$, obtaining the exponent sequence $<0001001000010000>$. As we know, to power this vector to the primes sequence is the same as to pick the primes corresponding to the non-zero entries and multiply them. Therefore, the integer referred to in the weight vector (in complementary mode) is $G_{D}=7$ $\times 19 \times 41=5453$. Given this, we can express the hi-hat code in the form $\mathrm{M}_{\mathrm{h}}=(16,-1,5453)$.

Finally, the codes of the individual pieces can be combined into a unique structure (denoted as $\underline{\mathrm{M}})$ representing the complete groove. Ultimately, $\underline{\mathrm{M}}$ will be expressed as the septuplet $\underline{\mathrm{M}}=(\mathrm{c}$, $\left.M_{b}, M_{s}, M_{h}\right)=\left(c,\left[G_{R}, G_{D}\right]_{b},\left[G_{R}, G_{D}\right]_{s},\left[\left[G_{R}, G_{D}\right]_{h}\right)\right.$. Considering our example, $M=(16,394174$, $4072315,2515590,-1,5453)$.

The retrieval of a three-piece drum groove, given a code $\mathrm{M}$, is achieved through the application of the same algorithm TranslateCode, used for isolated rhythms (see Figure 11). However, due to the compound structure of the groove-code, some new elements have to be incorporated in the definitive implementation of the algorithm. Firstly, the algorithm shall be able to go through $\mathrm{M}$ in a two-step window from the second entry on, in order to adequately process the three pairs of information, respectively related to any of the groove's streams. Furthermore, it is also necessary to provide the algorithm with the necessary conditions for dealing with eventual complementary rhythms, indicated by negative entries.

Before continuing, especially envisaging further improvement, let us assign variables to denote the seven entries of $\underline{M}$ (we arbitrarily chose for this task the sequence of the alphabet between letters $i$ and $o$ ). Thus, from now on a generic compound code will be denoted as $\underline{\mathrm{M}}=(\mathrm{i}, \mathrm{j}, \mathrm{k}, \mathrm{l}, \mathrm{m}$, $\mathrm{n}, \mathrm{o})$. Figure 15 illustrates the functioning of the definitive version of TranslateCode taking the code 
$\underline{\mathrm{M}}$ as input.

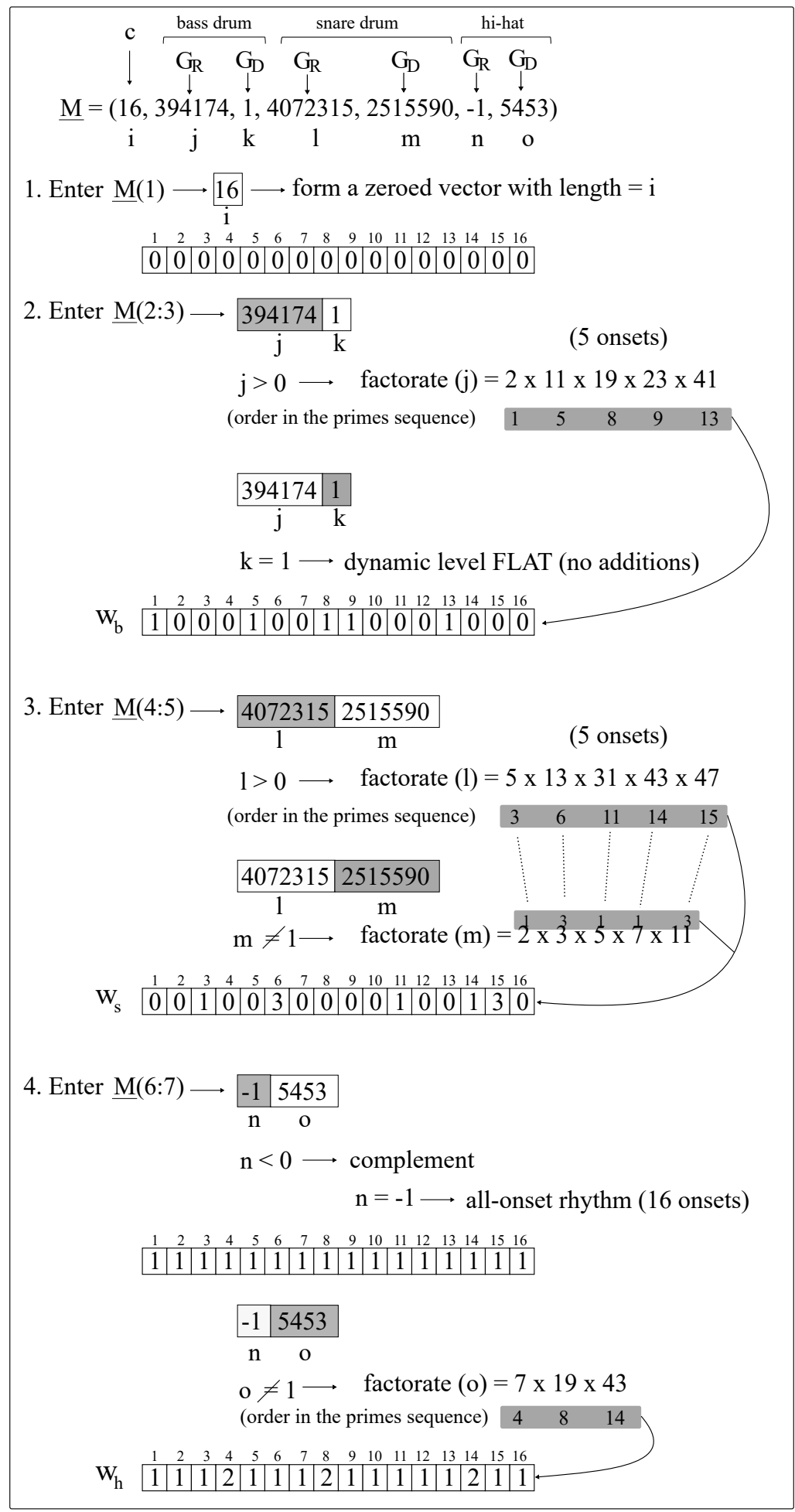

Figure 15: Basic algorithm of TranslateCode for recovering a three-stream groove rhythm $R$ from a $\underline{M}$ code. 


\section{ENCODING RHYTHMIC VARIATIONS}

Maybe the most attractive application of the encoding technology addresses the aspect of rhythmic variations, both with analytical and compositional purposes. For this, instead of dealing with the content of a given referential $\underline{M}$ expressed with actual numbers, we need only to use its algebraic version (i.e., denoted by variables i-o). ${ }^{19}$

Firstly, let us consider the entries associated uniquely with onset configurations (variables $\mathrm{j}, \mathrm{l}$, and $\mathrm{n})$. There are three manners of transforming an onset pattern of a rhythm $\mathrm{R}$ :

1. By adding a new onset to $\mathrm{R}$ - this is equivalent to multiplying the variable in question by a prime number of the sequence that is not a factor of $R$;

2. By deleting an existent onset - this is equivalent to dividing the variable in question by a prime number of the sequence that is already a factor of $\mathrm{R}$;

3. By replacing an existent onset by another one -- this is equivalent to multiplying the variable in question by a fraction such that the numerator is a new prime and the denominator is an already existing one, which is intended to be replaced.
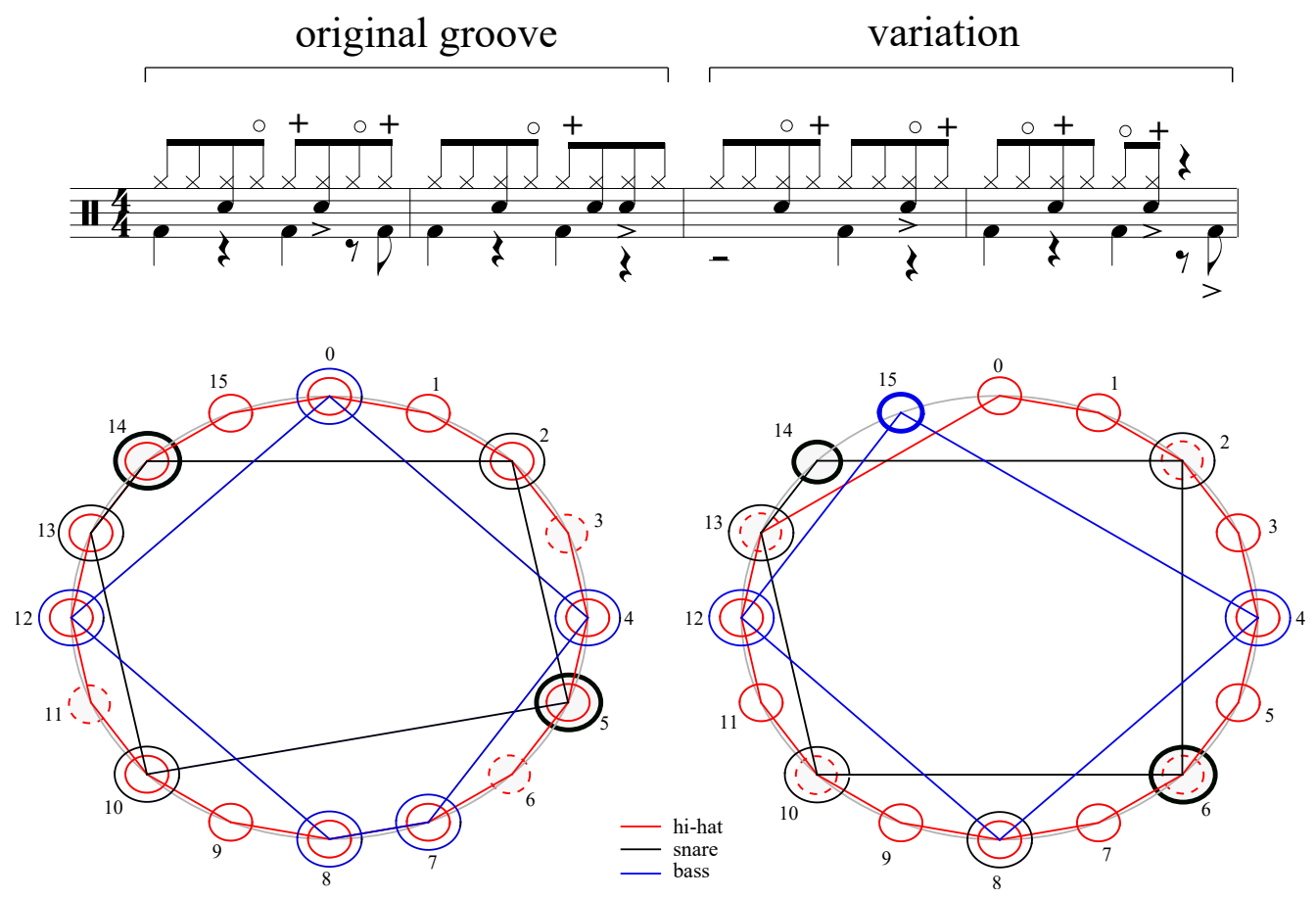

Figure 16: A possible variation of Figure 13.

In the case of the additional information of a groove (dynamics or timbre, represented by variables $\mathrm{k}, \mathrm{m}$, and $\mathrm{o}$ ), transformations are referred to a unique category, namely the change of a dynamic level or a timbre of a specific onset, which is achieved by multiplying or dividing the

\footnotetext{
${ }^{19}$ Notice that if we consider $\underline{M}$ algebraically, it is not necessary any more to replace an eventual very intense rhythm by its complement. In this manner, considering our example, variable $n$ (that denotes the hi-hat onset configuration) represents the original (very huge) integer which corresponds to the product of the sixteen first prime numbers, rather than its complement $(-1)$.
} 
variable in question by a prime factor related to the original configuration (such procedure affects the magnitude of the exponents, by adding or subtracting units). Finally, the cycle itself (variable i) can also be transformed, by expansion or contraction, by multiplication and/or division. ${ }^{20}$

As an illustration of how these procedures can be applied in an analytical situation, consider the variation of our original groove shown in Figure 16. As above stated, let the groove be expressed as $\underline{M}=(i, j, k, l, m, n, o)$. Given this, it is possible analyze the variant, whose code we will denote as $\underline{\mathrm{M}}_{\mathrm{V}}$, in terms of algebraic transformations of the content of $\underline{\mathrm{M}}$.

For clarity, Table 3 details the transformational process, considering the individual vector differences between both compound rhythms. Bold-face numbers indicate affected onsets or dynamic/timbral elements.

Table 3: Transformations involved in the variation of Figure 16

\begin{tabular}{cccccc}
\hline \hline & & groove & variation & divide by & multiply by \\
\hline cycle & $\mathrm{i}$ & 16 (scalar) & 16 (scalar) & 1 & 1 \\
\hline bass & $\mathrm{j}$ & $\mathbf{1 0 0 0 1 0 0 1 1 0 0 0 1 0 0 0}$ & $\mathbf{0 0 0 0 1 0 0 0 1 0 0 0 1 0 0 1}$ & 19 & 53 \\
drum & $\mathrm{k}$ & 11111 & 11113 & 1 & $11^{2}$ \\
\hline snare & $\mathrm{l}$ & $00100 \mathbf{1 0 0 0 0 1 0 0 1 1 0}$ & 0010001000100100 & $13 \times 47$ & 17 \\
drum & $\mathrm{m}$ & 13113 & 13130 & $11^{3}$ & $7^{2}$ \\
\hline hi-hat & $\mathrm{n}$ & 1111111111111111 & 1111111111111100 & $47 \times 53$ & 1 \\
& $\mathrm{o}$ & 1112112111121111 & 1121112112111100 & $7 \times 37 \times 47 \times 53$ & $5 \times 23$ \\
\hline
\end{tabular}

This allows us to propose the following algebraic representation of the variation:

$$
\underline{\mathrm{M}}_{\mathrm{V}}=\left(\mathrm{i}, \frac{19}{53} \mathrm{j}, \frac{1}{11^{2}} \mathrm{k}, \frac{611}{17} \mathrm{l}, \frac{11^{3}}{7^{2}} \mathrm{~m}, 2491 \mathrm{n}, \frac{645169}{115} \mathrm{o}\right)
$$

Let us now examine another very useful application. Consider an archetypal samba drum groove, which is characterized by a fixed bass-drum pattern (normally doubling rhythmically the bass line) over which cross-stick attacks and ride accents are added in almost random sixteenthnote onsets. In a sense, most of samba swing lies precisely in the low expectancy of these accentuation patterns. Normally, the decisions about where and when an onset shall be stressed depends on the drummer's intuition and, of course, expertise in playing this genre of music, in sum, of his/her improvisatory capacity in this kind of music. Now, suppose that we intend to model a samba-drum performance, as in the excerpt shown in Figure 17. Instead of treating the whole passage as a 40-pulse cycle, which would turn the underlying computation highly complex and costly, an alternative, much simpler strategy is to see it as a set of nine variations of a very small basic "theme", at the first beat of the groove. According to this approach, it can be seen as the generator of the nine subsequent transformed versions of itself. Notice that now generator and variants are quite small cycles (four pulses), which implies few and small primes $(2,3,5$, and 7 ), and considerably simple codes. Observe also that five out seven structures are kept invariant along the groove: cycle length (i), bass-drum onset and dynamic/timbral configurations ( $\mathrm{j}-\mathrm{k})$, snare dynamic/timbral pattern (m), and ride onset positions (n). As a matter of fact, cross-stick positions (l) and ride accentuations (o) are the only parameters subject to variation.

Table 4 depicts the algebraic structure of the nine variations in relation to that of the generator (invariant elements are let shaded). Observe that, although variation 4 incidentally replicates the

\footnotetext{
${ }^{20}$ For example, the transformation of a 16-pulse into a 12-pulse cycle is achieved through the multiplication of $i$ by $\frac{3}{4}$
} 


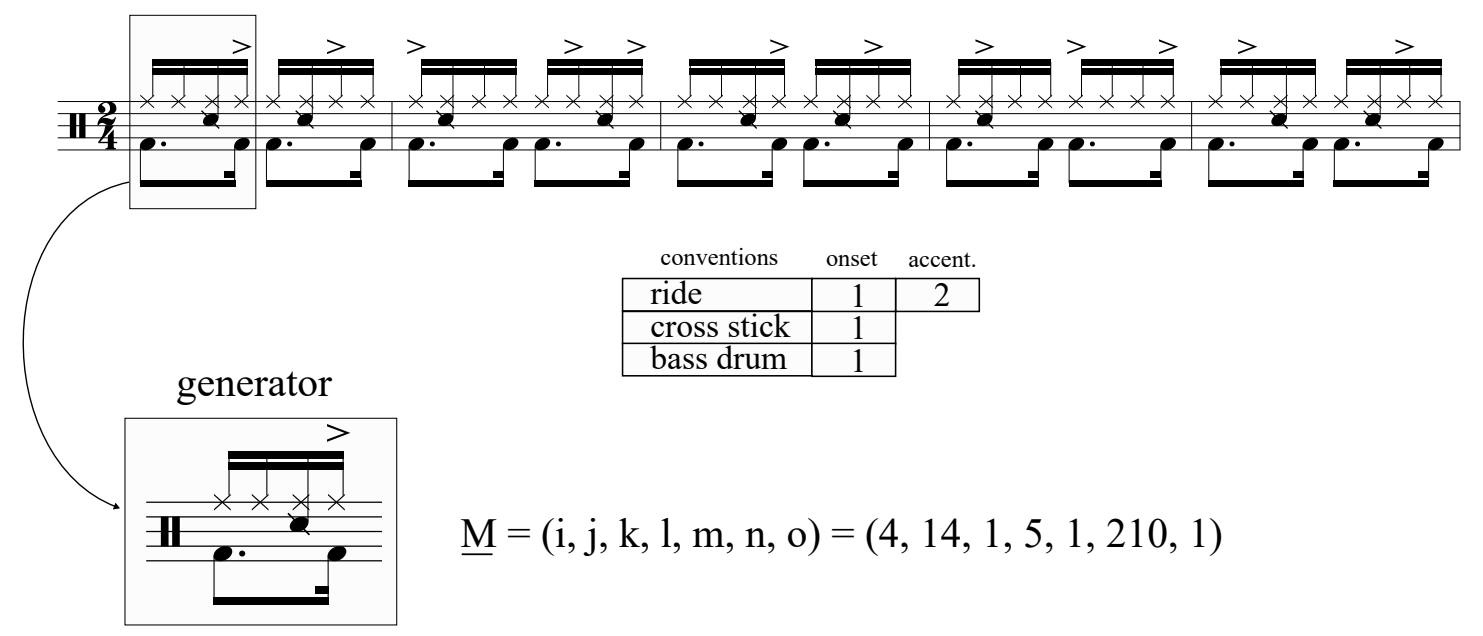

Figure 17: Samba variations by transformations applied to a basic generator.

generator (which is perfectly normal in real-music situation), there is a considerable variety of patterns among the remaining variants, due to combinatorial possibilities.

Table 4: Transformations involved in the variation of Figure 16

\begin{tabular}{llllllllc}
\hline \hline $\mathrm{m} .1$ & generator & $\mathrm{i}$ & $\mathrm{j}$ & $\mathrm{k}$ & $\mathrm{l}$ & $\mathrm{m}$ & $\mathrm{n}$ & $\mathrm{o}$ \\
& variant 1 & 1 & 1 & 1 & $\frac{3}{5} \mathrm{l}$ & 1 & 1 & $\frac{5}{7} \mathrm{o}$ \\
\hline $\mathrm{m} .2$ & variant 2 & 1 & 1 & 1 & $\frac{3}{5} \mathrm{l}$ & 1 & 1 & $\frac{2}{7} \mathrm{o}$ \\
& variant 3 & 1 & 1 & 1 & 1 & 1 & 1 & $3 \mathrm{o}$ \\
\hline $\mathrm{m} .3$ & variant 4 & 1 & 1 & 1 & 1 & 1 & 1 & 1 \\
& variant 5 & 1 & 1 & 1 & $\frac{3}{5} \mathrm{l}$ & 1 & 1 & $\frac{5}{7} \mathrm{o}$ \\
\hline $\mathrm{m} .4$ & variant 6 & 1 & 1 & 1 & $\frac{3}{5} \mathrm{l}$ & 1 & 1 & $\frac{3}{7} \mathrm{o}$ \\
& variant 5 & 1 & 1 & 1 & $\frac{1}{5} \mathrm{l}$ & 1 & 1 & $2 \mathrm{o}$ \\
\hline $\mathrm{m} .5$ & variant 7 & 1 & 1 & 1 & 1 & 1 & 1 & $\frac{3}{7} \mathrm{o}$ \\
& variant 8 & 1 & 1 & 1 & $\frac{3}{5} 1$ & 1 & 1 & $\frac{5}{7} \mathrm{o}$ \\
\hline
\end{tabular}

Lastly, we can express the whole passage as a $10 \times 7$ matrix, in which any row represents a beat (the generator and the nine variants) and the columns correspond to the elements of the respective $\mathrm{M}$ codes.

$$
\left|\begin{array}{ccccccc}
1 & 1 & 1 & 1 & 1 & 1 & 1 \\
1 & 1 & 1 & 3 / 5 & 1 & 1 & 5 / 7 \\
1 & 1 & 1 & 3 / 5 & 1 & 1 & 2 / 7 \\
1 & 1 & 1 & 1 & 1 & 1 & 3 \\
1 & 1 & 1 & 1 & 1 & 1 & 1 \\
1 & 1 & 1 & 3 / 5 & 1 & 1 & 5 / 7 \\
1 & 1 & 1 & 3 / 5 & 1 & 1 & 3 / 7 \\
1 & 1 & 1 & 1 / 5 & 1 & 1 & 2 \\
1 & 1 & 1 & 1 & 1 & 1 & 3 / 7 \\
1 & 1 & 1 & 3 / 5 & 1 & 1 & 5 / 7
\end{array}\right|
$$




\section{CONCLUSION}

This article introduced an original theoretical-methodological proposal intended to address drumset rhythms in a formal manner. The adaptation and combination of concepts, formulations, and conventions coined by Gauss, Gödel, and Toussaint that resulted in the technology which is here described allows complex three-stream drum grooves to be represented both geometrically (as superimposed polygons) and algebraically (as tuples of codes, vectors, and matrices). By exploring the inherent property of prime numbers (expressed in the FTA), some simple algorithms are used for plotting and encoding rhythms of the individual pieces of a groove, as well as for the reversal process, retrieving the original timelines by factoring their codes. Our proposal includes also the possibility of extending the methodology to encompass auxiliary parameters, as dynamics and timbre, turning the model more flexible and comprehensive. Besides the primary advantages of encoding relatively complex musical information as retrievable tuples (compactness, especially), the practical applications that are here envisaged - both in analysis and composition - open a potentially wide avenue to be explored in future work. In this sense, the idea of using this technology for creating subtle variations of a groove-seed (expressed as an abstract generator), as it was done in the samba example, seems to be the most promising.

\section{REFERENCES}

[1] Almada, C. 2017. Gödel-vector and Gödel-address as Tools for Genealogical Determination of Genetically-Produced Musical Variants. In: Pareyon, D.; Pina-Romero, S., Agustín-Aquino, G.; Lluis-Plueba, E. (eds.) The Musical-Mathematical Mind: Patterns and Transformations. Cham (Switzerland): Springer Verlag, pp. 9-16.

[2] Cohn, R. 1992a. The Dramatization of Hypermetric Conflicts in the Scherzo of Beethoven's Ninth Symphony. 19th-Century Music, v. 15, n. 3, pp. 188-206.

[3] Cohn, R. 1992b. Metric and Hypermetric Dissonance in the Menuetto of Mozart's G-Minor Symphony, K.550. Intégral v. 6, pp. 1-33.

[4] Cohn, R. 2001. Complex Hemiolas, Ski-Hill Graphs and Metric Spaces. Music Analysis, v. 20, n. 3, pp. 295-326.

[5] Cohn, R. 2016. A Platonic Model for Funky Rhythms. Music Theory Online, v. 22, n. 2. Available at: https://mtosmt.org/issues/mto.16.22.2/mto.16.22.2.cohn.php.

[6] Figueiredo, A.; Wolf, P. S. A. 2009. Assortative Pairing and Life History Strategy - A Cross-Cultural Study. Human Nature, v. 20, pp. 317-330.

[7] Gotham, M. 2005. Attractor Tempos for Metrical Structures. Journal of Mathematics and Music, v. 9, n. 1, pp. 23--44.

[8] Gómez-Martín, F.; Taslakian, P.; Toussaint, G. 2009. Interlocking and Euclidean Rhythms. Journal of Mathematics and Music, v. 3, n. 1, pp. 15--30.

[9] Guastavino, C.; Gómez F.; Toussaint, G.; Marandola, F.; Gómez, E. 2009. Measuring Similarity between Flamenco Rhythmic Patterns. Journal of New Music Research, v. 38, n. 2, pp. 129--38.

[10] Guerra, S. 2018. Expanded Meter and Hemiola in Baden Powell's Samba-Jazz. Dissertation (PhD in Music), Yale University. 
[11] Guerra, S. 2018. Toward a Theory of Structuring Rhythm in Improvisation in Timeline-Based Musics. MusMat: Brazilian Journal of Music and Mathematics, v. 3, n. 1, pp. 44--60.

[12] Hardesty, J. 2016. A Self-Similar Map of Rhythmic Components. Journal of Mathematics and Music, v. 10, n. 1, pp. 36--58.

[13] Herrlein, J. 2018. Das alturas ao ritmo: teoria dos conjuntos rítmicos como ferramenta composicional. Dissertation (PhD in Music), Federal University of Rio Grande do Sul.

[14] Hoesl, F.; Senn, O. 2018. Modelling Perceived Syncopation in Popular Music Drum Patterns: A Preliminary Study. Music \& Science, v. 1, pp. 1--15.

[15] London, J. 2002. Some Non-Isomorphisms between Pitch and Time. Journal of Music Theory v. 46, n. 1-2, pp. 127--51.

[16] Murphy, S. 2016. Cohn's Platonic Model and the Regular Irregularities of Recent Popular Multimedia. Music Theory Online, v. 22, n. 3. Available at: https://mtosmt.org/issues/mto.16.22.3/mto.16.22.3.murphy.html

[17] Nagel, E.; Newman, J. 2001. Gödel 's Proof. New York: New York University Press.

[18] Temperley, D. 2001. The Cognition of Basic Musical Structures. Cambridge: The MIT Press.

[19] Toussaint, G. 2013. The Geometry of Musical Rhythm: What Makes a "Good" Rhythm Good? Boca Raton: CRC Press. 